\title{
Mic60/Mitofilin determines MICOS assembly essential for mitochondrial dynamics and mtDNA nucleoid organization
}

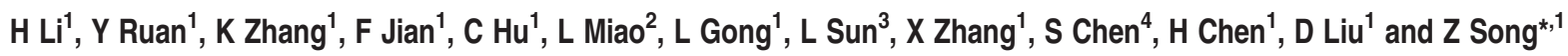

The MICOS complex (mitochondrial contact site and cristae organizing system) is essential for mitochondrial inner membrane organization and mitochondrial membrane contacts, however, the molecular regulation of MICOS assembly and the physiological functions of MICOS in mammals remain obscure. Here, we report that Mic60/Mitofilin has a critical role in the MICOS assembly, which determines the mitochondrial morphology and mitochondrial DNA (mtDNA) organization. The downregulation of Mic60/Mitofilin or Mic19/CHCHD3 results in instability of other MICOS components, disassembly of MICOS complex and disorganized mitochondrial cristae. We show that there exists direct interaction between Mic60/Mitofilin and Mic19/CHCHD3, which is crucial for their stabilization in mammals. Importantly, we identified that the mitochondrial i-AAA protease Yme1L regulates Mic60/Mitofilin homeostasis. Impaired MICOS assembly causes the formation of 'giant mitochondria' because of dysregulated mitochondrial fusion and fission. Also, mtDNA nucleoids are disorganized and clustered in these giant mitochondria in which mtDNA transcription is attenuated because of remarkable downregulation of some key mtDNA nucleoid-associated proteins. Together, these findings demonstrate that Mic60/Mitofilin homeostasis regulated by Yme1L is central to the MICOS assembly, which is required for maintenance of mitochondrial morphology and organization of mtDNA nucleoids.

Cell Death and Differentiation (2016) 23, 380-392; doi:10.1038/cdd.2015.102; published online 7 August 2015

Mitochondria have a key role in oxidative phosphorylation and related cellular metabolism, in energy conversion, in programmed cell death, in cell growth and in diseases. Mitochondrial outer and inner membranes strongly differ in architecture and functions. The mitochondrial outer membrane forms a barrier to cytosol, and contains channels and the translocases of outer membrane, which is the main protein entry gate of mitochondria. ${ }^{1,2}$ In contrast, the mitochondrial inner membrane consists of two morphologically distinct regions: the inner boundary membrane is in close proximity to the outer membrane and the cristae membranes that are large tubular invaginations. ${ }^{3-8}$ The mitochondrial inner boundary and cristae membrane are physically separated by cristae junctions, which are narrow tubular or slot-like structure. ${ }^{4,9}$

The mitochondrial cristae are arranged in regular arrays and are the main sites of ATP production in the mitochondria, but the molecules that are associated with the maintenance of cristae architecture still remain elusive. Recently, several groups identified a large protein complex, MICOS complex (mitochondrial contact site and cristae organizing system; previously named MINOS, MitOS, Mitofilin or Fcj1 complex ), that has a crucial role in the formation of cristae junctions, contact sites to the outer membrane, and the organization of inner membrane. ${ }^{10-14}$ In yeast, MICOS consists of at least six subunits: Mic60 (Fcj1), Mic10 (Mio10/Mcs10/Mos1), Mic19 (Aim13/Mcs19), Mic26 (Mio27/Mcs29/Mos2), Mic12 (Aim5/ Msc12) and Mic27 (Aim37/Mcs27). In mammals, five subunits of MICOS have already been identified, including Mic60 (Mitofilin/IMMT), Mic10 (MINOS1), Mic19 (CHCHD3/MINOS3), Mic25 (CHCHD6/CHCM1) and Mic27 (APOOL). ${ }^{15-18}$ However, the physiological functions of MICOS and how MICOS is assembled in mammals are largely unknown.

The maintenance of mitochondrial protein homeostasis is critical for mitochondrial functions. Mitochondrial inner membrane i-AAA protease $Y m e 1 \mathrm{~L}$ is a key regulator in the quality control of mitochondrial proteins. ${ }^{19}$ Yme1L exerts ATPdependent proteolytic activity, resulting in either degradation or processing of its substrates such as optic atrophy 1 (OPA1) and some subunits of oxidative phosphorylation. ${ }^{20-22}$

Mitochondria are dynamic organelles whose morphology are determined by continuous fusion and fission events. ${ }^{23}$ Mfn1, Mfn2 and OPA1 are key factors for mitochondrial fusion; ${ }^{24}$ dynamin-related protein 1 (Drp1) is a cytosolic protein and is essential for mitochondrial fission, it is recruited by mitochondrial outer membrane protein mitochondrial fission factor (Mff), mitochondrial dynamics protein $49 \mathrm{kDa}$

${ }^{1}$ College of Life Sciences, Wuhan University, Room 4122, Wuhan 430072, PR China; ${ }^{2}$ Scientific and Educational Department, Second Hospital of AnHui Medical University, 678 Furong Road, Hefei 230601, PR China; ${ }^{3}$ State Key Laboratory of Medical Molecular Biology, Department of Biochemistry and Molecular Biology, Institute of Basic Medical Sciences, Chinese Academy of Medical Sciences, Peking Union Medical College, Beijing, PR China and ${ }^{4}$ School of Pharmaceutical Sciences, Wuhan University, Wuhan 430072, PR China

*Corresponding author: Z Song, College of Life Sciences, Wuhan University, Room 4122, Wuhan 430072, PR China. Tel: +86-27-68752235; Fax: 86-551-63606264; E-mail: songzy@whu.edu.cn

Abbreviations: MEF, mouse embryonic fibroblast; OPA1, optic atrophy 1; Drp1, dynamin-related protein 1; Mff, mitochondrial fission factor; MiD49/51, mitochondrial dynamics proteins of $49 \mathrm{kDa}$ and $51 \mathrm{kDa}$; WT, wild type; shMic60, short hairpin-mediated RNA interference to Mic60; mtDNA mitochondrial DNA; co-IP co-immunoprecitation; MICOS mitochondrial contact site and cristae organizing system

Received 10.12.14; revised 14.5.15; accepted 22.6.15; Edited by L Scorrano; published online 07.8.15 
(Mid49) and Mid51 during fission. ${ }^{25}$ Mitochondrial dynamics are crucial for normal mitochondrial network and cellular functions, ${ }^{26}$ and are highly associated with some neurodegenerative diseases. ${ }^{27,28}$ The giant mitochondria, which are enlarged spherical mitochondria, were rare in normal cells, but were observed more frequently in aged or diseased cells. ${ }^{29-33}$ Yeast cells lacking Mdm10, Mdm12, Mdm1, Mdm31 or Mdm32 harbor giant mitochondria with abnormal internal structure and mitochondrial DNA (mtDNA) nucleoids; ${ }^{34-37}$ however, no mammalian homologs of Mdm10, Mdm12, Mdm1, Mdm31 or Mdm32 are identified, and the functions and molecular mechanisms for the formation of giant mitochondria in mammals remain poorly understood.

Mitochondria contain mtDNA, which encodes several key components of oxidative phosphorylation. mtDNA is associated with several proteins and packaged into nucleoprotein complexes called 'mitochondrial nucleoids' or 'mtDNA nucleoids'. ${ }^{38,39}$ mtDNA nucleoids are crucial for the biogenesis and maintenance of mtDNA. ${ }^{40}$ mtDNA nucleoids are dynamic and relatively uniform in shape and size ${ }^{41}$ but the factors for controlling the distribution and organization of mtDNA nucleoids remain to be explored.

Mic60/Mitofilin, a key component of MICOS complex, was altered under several different pathological conditions. ${ }^{42-46}$ However, how the integrity of MICOS and the homeostasis of MICOS subunits are controlled and the physiological functions of MICOS complex in mammals still remain unknown. In the present study, we show that the stability of Mic60/Mitofilin is dependent on its assembly into MICOS complex by direct interaction with Mic19/CHCHD3, and mitochondrial protease Yme1L regulates Mic60/Mitofilin homosteosis. Moreover, we reveal that the integrity of MICOS complex is critical for the maintenance of mitochondrial morphology and the organization of mtDNA nucleoids.

\section{Results}

The deficiency of MICOS complex subunits leads to disorganized cristae structure. In mammals, Mic60 (Mitofilin/IMMT), Mic10 (MINOS1), Mic19 (CHCHD3/MINOS3) and Mic25 (CHCHD6) have been identified to be main subunits of MICOS complex. ${ }^{15-17}$ We then study the effect of the knockdown of Mic60/Mitofilin, Mic19/CHCHD3, Mic10/MINOS1, Mic25/CHCHD6 or Mic25/CHCHD6 plus Mic19/CHCHD3, respectively, on mitochondrial cristae architecture in mouse embryonic fibroblasts (MEFs) by transmission electron microscope. In shMic60 (short hairpin-mediated RNA interference to Mic60), shMic19 or shMic10 MEFs, most of mitochondrial cristae junctions were lost, but little onion-like cristae structure was found (Figure $1 \mathrm{~A}$ and Supplementary Figure 1A); in addition, mitochondrial cristae junctions were moderately reduced in shMic25 MEFs (Figure $1 \mathrm{~A}$ and Supplementary Figure 1A). In addition, comparing with wild type (WT), shMic60, shMic19, shMic10 but not shMic25 mitochondria were found to harbor reduced cristae (Figure $1 \mathrm{~A}$ and Supplementary Figure 1B), indicating that Mic60/Mitofilin, Mic19/CHCHD3 and Mic10 are essential for the formation of mitochondrial cristae junctions and have an important role in maintenance of mitochondrial cristae structure.

Mic25/CHCHD6 is highly homologous with Mic19/CHCHD3 in mammalian, ${ }^{17}$ we then examined the effect of Mic25 and Mic19 double knockdown on mitochondrial cristae structure. As shown in Figure 1A,Supplementary Figures $1 \mathrm{~A}$ and $\mathrm{B}$, shMic25 slightly reduced mitochondrial cristae junctions, and Mic25 and Mic19 double knockdown have the similar effect on the loss of mitochondrial cristae or cristae junctions with shMic19, suggesting that Mic19/CHCHD3 is more critical than Mic25/CHCHD6 in organizing mitochondrial cristae structure.

Mic60/Mitofilin and Mic19/CHCHD3 homeostasis are essential for the integrity of MICOS complex in mammalian cells. To analyze the MICOS complex homeostasis, we check the effect of Mic60/Mitofilin, Mic19/CHCHD3, Mic10/MINOS1 or Mic25/CHCHD6 knockdown on the steady-state protein levels of other MICOS complex subunits. As shown in Figure $1 \mathrm{~B}$, shMic60 results in remarkable downregulation of Mic19/CHCHD3, Mic10/MINOS1 and Mic25/CHCHD6 (Figure 1B-a); similarly, shMic19 also leads to dramatical reduction of Mic60/Mitofilin and Mic10/MINOS1, but has no effect on stability of Mic25/CHCHD6 (Figure 1B-b), suggesting that the steady-state levels of Mic60/Mitofilin and Mic19/CHCHD3 are highly correlated. However, the levels of Mic60/Mitofilin, Mic19/CHCHD3 and Mic25/CHCHD6 were unchanged in shMic10 cells (Figure 1B-c). In addition, Mic60/Mitofilin was slightly reduced but Mic19/CHCHD3 and Mic10/MINOS1 remain unchanged in shMic25 cells (Figure 1B-d), suggesting that Mic25/CHCHD6 is partially associated with Mic60/Mitofilin proteolysis. Taken together, Mic60/Mitofilin and Mic19/CHCHD3 are critical for stability of MICOS complex subunits.

It is still unknown how MICOS complex is organized in mammals. To test the role of Mic60/Mitofilin or Mic19/CHCHD3 in assembly of MICOS complex, mitochondria from WT, shMic10, shMic25, shMic19 or shMic60 MEFs were separated by BN-PAGE and then analyzed by western blot analysis. As shown in Supplementary Figure 1C, the similar size of MICOS complex were detected by western blot using anti-Mic60, antiMic19 or anti-Mic10 antibodies, respectively, suggesting that Mic60/Mitofilin, Mic19/CHCHD3 and Mic10/MINOS1 are integrated into MICOS complex. Importantly, shMic60 or shMic19 results in remarkable reduced MICOS complex (Figure 1C); however, shMic10 leads to slight decrease in MICOS complex in MEFs (Figure 1C). These results demonstrate that both Mic60/Mitofilin and Mic19/CHCHD3 are crucial for the integrity of MICOS complex. In addition, shMic25 causes medium reduction of MICOS complex in MEFs (Figure 1C); the reduction of MICOS complex induced by shMic25 is possible due to the slight reduction of Mic60 (Figure 1B-d).

To explore whether the stability of mammalian MICOS subunits are dependent on their status of assembly, we infected HeLa cells with lentivirus containing pMSCV/Mic60Flag, pMSCV/Mic19-Flag or pMSCV/Mic10-Flag. BN-PAGE and western blot analysis revealed that exogenous expressed Mic60-Flag, Mic19 -Flag or Mic10-Flag could be assembled into MICOS complex (Supplementary Figure 1C), and the expression of exogenous MICOS subunits results in the 
A

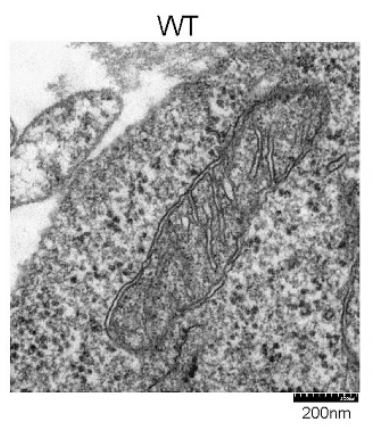

shMic10

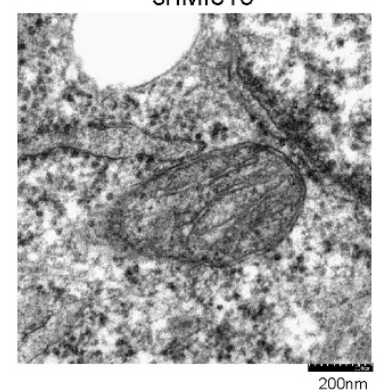

B

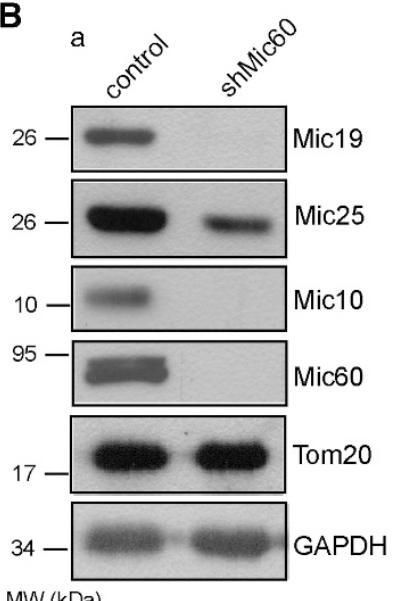

C

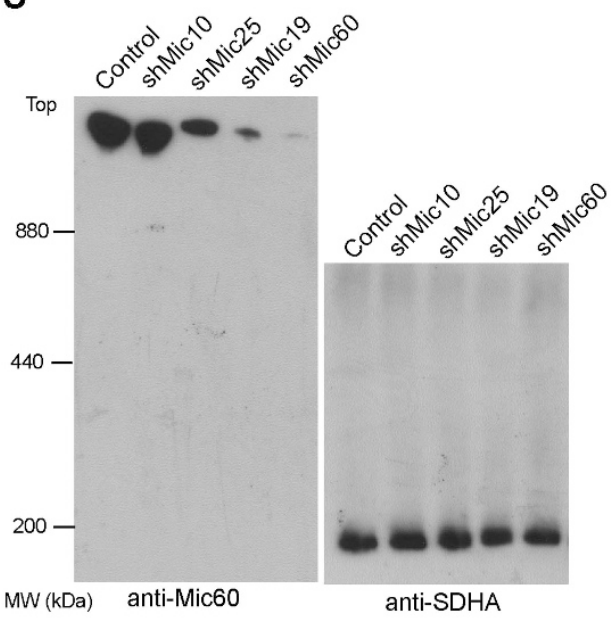

shMic60

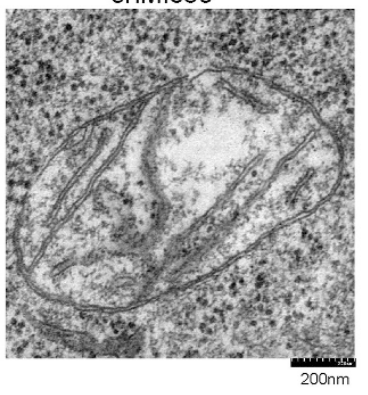

shMic25

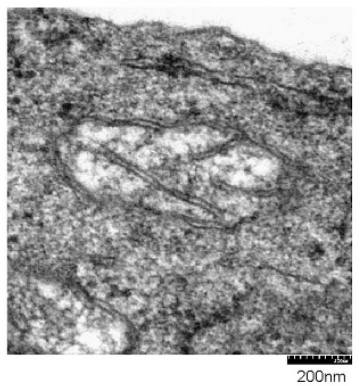

b

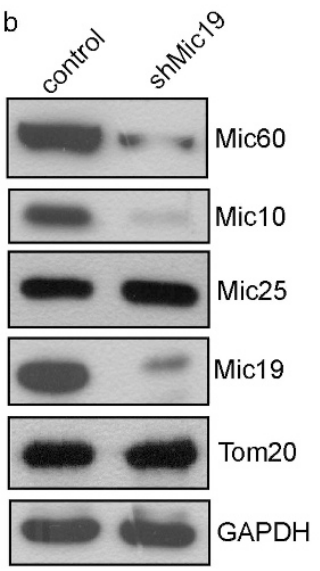

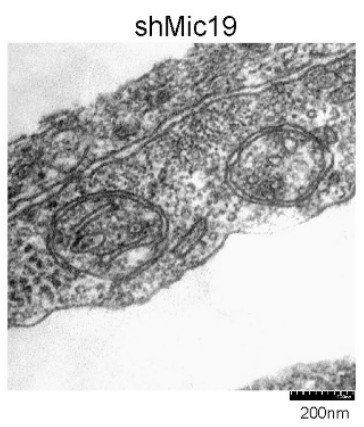

shMic25 +shMic19

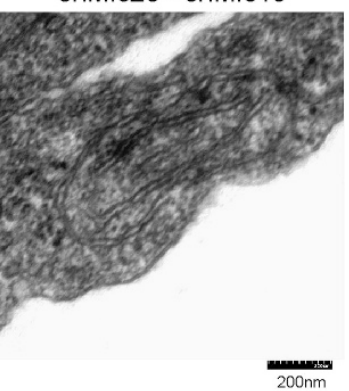

C

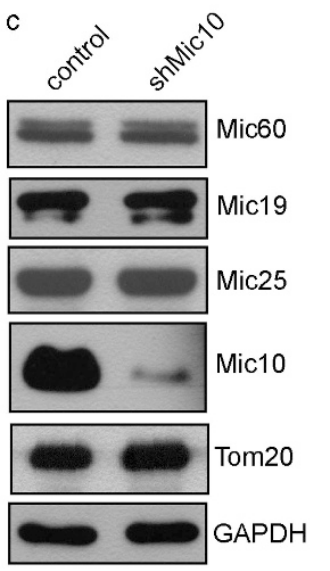

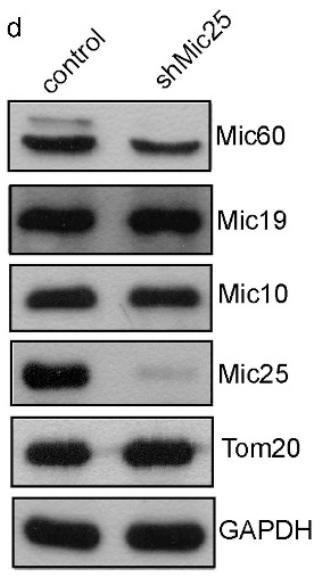

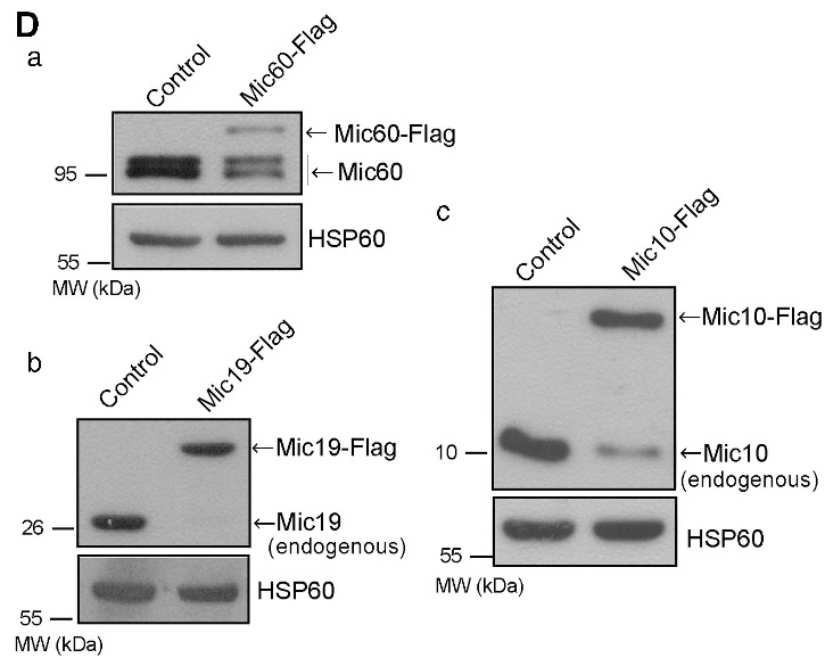


decrease or disappearance of the corresponding endogenous proteins in HeLa cells (Figure 1d). In addition, the total exogenous and endogenous protein levels are equal to the level of endogenous protein in control cells (Figure 1D). These results suggest that unassembled Mic60/Mitofilin, Mic19/CHCHD3 or Mic10/MINOS1 are unstable and degrade quickly. We also found that mitochondrial morphology is not changed upon co-expression of Mic60-GFP and Mic19-Flag in HeLa cells, and Mic60-GFP is not increased when coexpressed with Mic19-Flag (Supplementary Figures 2A and B), indicating that besides Mic19/CHCHD3, some other MICOS subunits are still required for the stability of Mic60/Mitofilin.

Mic60/Mitofilin directly interacts with Mic19/CHCHD3. Mic60/Mitofilin harbors two coiled coil domains, and Mic19/CHCHD3 has a CHCH domain (Figure 2A). We check the relationship between Mic60/Mitofilin and Mic19/CHCHD3 by co-immunoprecipitation (co-IP) assay. Figure 2B-a showed that Mic19-Flag but not beads alone (control) successfully precipitated Mic60-GFP, indicating that Mic19/ CHCHD3 binds to Mic60/Mitofilin. In addition, we used antiMic60 antibody to perform co-IP assay in HeLa cells, and found that the endogenous Mic60/Mitofilin interacts with endogenous Mic19/CHCHD3 (Figure 2B-b). We also expressed recombinant GST, GST-Mic60(35-200), GSTMic60(35-480), GST-Mic60(371-590) or Mic19-His in E. coli to perform GST pull-down assay in vitro. As shown in Figure 2c, Mic19-His was precipitated with GST-Mic60(371$590)$ but not with GST, GST-Mic60(35-200) or GST-Mic60 (35-480), suggesting that GST-Mic60(371-590) interacts directly with Mic19-His. Taken together, the interaction between Mic60/Mitofilin and Mic19/CHCHD3 is direct, and the region binding to Mic19/CHCHD3 locates at residues $371-590$ of Mic60/Mitofilin.

Mitochondrial i-AAA protease Yme1L regulates Mic60/ Mitofilin homeostasis. Mitochondrial i-AAA protease Yme1L locates at mitochondrial inner membrane and regulates quality control of mitochondrial proteins. ${ }^{19-22}$ To clarify how Mic60/Mitofilin is degraded in mitochondria, we check the relationship between Yme1L and Mic60/Mitofilin. As WT Yme1L interacts and reacts with its substrates transiently, we mutated the glutamate residue of $\mathrm{HEXXH}$ motif, which is required for proteolysis in human $Y$ me1L to glutamine (E600Q), and the mutant Yme1L-E600Q binds to its substrates stably. As show in Figure 3A-a, Yme1L-E600QMyc successfully precipitated Mic60-GFP but beads alone (control) did not, indicating that Yme1L interacts with Mic60/ Mitofilin. To further confirm the interaction, we used anti-Mic60 antibody to perform co-IP assay in HeLa cells, and endogenous Yme1L was detected in the precipitates
(Figures 3A-b), suggesting that endogenous Yme1L binds to endogenous Mic60/Mitofilin.

We also identified the region of Mic60/Mitofilin interacting with Yme1L by GST pull-down assay in vitro. Recombinant Mic60(371-590)-His was remarkably precipitated with GSTYme1L(46-773) (Figure 3B); in contrast, Mic60(35-200)-His or Mic60(35-480)-His was not detected in the precipitates with GST-Yme1L(46-773) (Figure 3B-b), suggesting that Mic60/Mitofilin directly interacts with Yme1L, and the binding region of Mic60/Mitofilin interacting with $\mathrm{Yme} 1 \mathrm{~L}$ is residues $371-590$, which is the same region binding to Mic19/CHCHD3.

To investigate the effect of Yme1L knockdown (shYme1L) on the protein level of Mic60/Mitofilin, the western blot and immunostaining analysis were performed. We revealed that the protein level of endogenous Mic60/Mitofilin was not altered in shYme1L MEFs (Figure 3C). However, loss of Mic19/ $\mathrm{CHCHD} 3$ largely reduced the protein level of Mic60/Mitofilin (Figure 3D), and the loss of $\mathrm{Yme1L}$ in shMic19 MEFs could rescue the protein level of Mic60/Mitofilin (Figure 3D). These results demonstrate that Mic60/Mitofilin is a proteolytic substrate of Yme1L under certain cellular conditions such as Mic19/CHCHD3 depletion, and indicate that Mic19/CHCHD3 may stabilize Mic60/Mitofilin by direct interaction to cover the binding site (Mic60(371-590)) of Mic60/Mitofilin and prevent Mic60/Mitofilin to be recognized and proteolysed by Yme1L under normal condition.

We also examined whether endogenous Mic60/Mitofilin are more stable in shYme1L cells after overexpression of Mic60Flag. We stably overexpressed Mic60-Flag in MEFs and then deleted Yme1L. We found that both the levels of exogenous Mic60-Flag and endogenous Mic60/Mitofilin in shYme1L MEFs are slightly more stable than the levels in Mic60-Flag overexpressed MEFs (Supplementary Figure 3A), suggesting that $\mathrm{Yme1L}$ has a role in regulating Mic60/Mitofilin degradation. In addition, shYme1L alone does not lead to the increase of endogenous Mic60/Mitofilin (Figure 3C-a), indicating that $\mathrm{Yme1L}$ is not only mitochondrial protease controlling Mic60/Mitofilin degradation and some other mitochondrial proteases may also regulate Mic60/Mitofilin proteolysis.

We also investigated the localization of Yme1L and Mic60/Mitofilin by immunostaining analysis. As shown in Supplementary Figure 3B, Mic60/Mitofilin is colocalized with Yme1L in the mitochondria.

Consistent with previous report, ${ }^{47}$ our data show that the reduction of Mic60/Mitofilin results in the loss of mitochondrial cristae junctions (Figure 1A). As Mic60/Mitofilin is substrate of $Y m e 1 L$, we then analyzed the mitochondrial ultrastructure in shYme1L MEFs by transmission electron microscope. Mitochondria in WT cells have well-defined lamellar cristae structures, whereas the majority of mitochondria in shYme1L cells display clearly abnormal cristae

Figure 1 Mic60/Mitofilin and Mic19/CHCHD3 are required for the structure of mitochondrial cristae and the assembly of MICOS complex. (A) Mitochondrial ultrastructure in WT, shMic60, shMic19, shMic10, shMic25 or shMic25 plus shMic19 MEFs were analyzed by transmission electron microscope. (B) Western blot analysis of Mic60/Mitofilin, Mic19/CHCHD3, Mic10/MINOS1 and Mic25/CHCHD6 in control, shMic60 (a), shMic19 (b), shMic10 (c) or shMic25 (d) MEFs. TOM20 and GAPDH served as protein-loading control. (C) Equal mitochondrial protein samples from control, shMic10, shMic25, shMic19 or shMic60 MEFs were separated by BN-PAGE, and MICOS complex were analyzed by western blot analysis with antibody against Mic60/Mitofilin. SDHA served as loading control. (D) Lysates form HeLa cells expressing Mic60-Flag (a), Mic19-Flag (b) or Mic10Flag (c) were analyzed by western blot analysis with antibodies against the indicated proteins 
structures (Supplementary Figure 3C), suggesting that $\mathrm{Yme1} \mathrm{L}$ is linked to maintaining mitochondrial cristae morphology. However, it is still obscure how Yme1L regulates mitochondrial ultrastructure. OPA1 is associated with mitochondrial cristae remodeling; ${ }^{48}$ our observations that both Mic60/Mitofilin and OPA1 are the substrates of Yme1L, ${ }^{20}$ provide important clues to study the role of $\mathrm{Yme1L}$ in regulating mitochondrial ultrastructure.
Downregulation of Mic60/Mitofilin causes giant mitochondria and impaired mitochondrial fusion and fission. To examine the role of MICOS complex in mitochondrial dynamics, we disrupted MICOS complex by knockdown of Mic60/Mitofilin, which stabilizes other MICOS components and is essential for integrity of MICOS (Figures 1B and C). Mitochondrial morphology was then assessed by confocal microscope in cells stably expressing

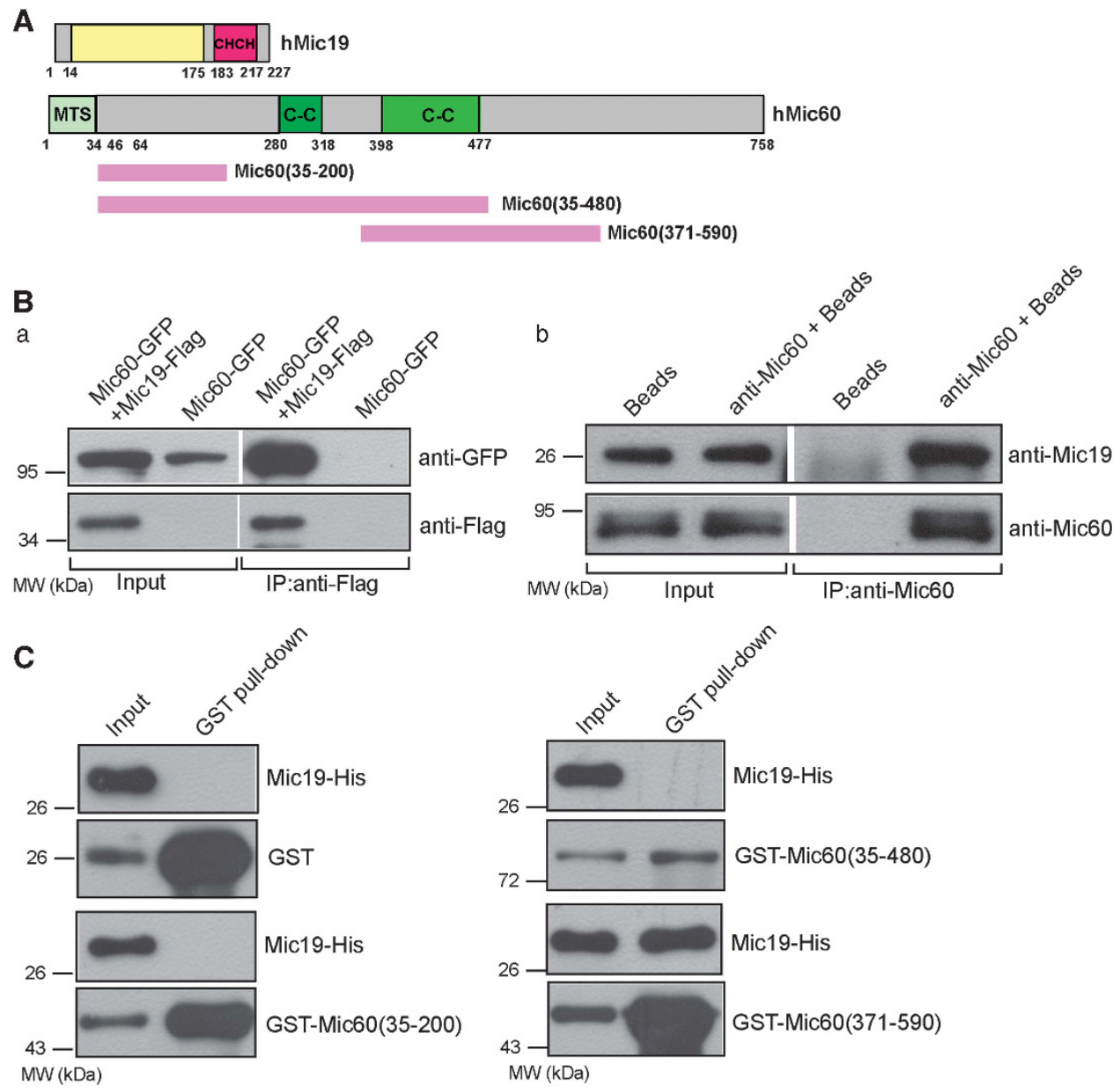

Figure 2 Mic19/CHCHD3 directly binds to Mic60/Mitofilin at the residues 371-590. (A) The schematic representation of human Mic19/CHCHD3 and Mic60/Mitofilin. 'MTS' indicates 'mitochondrial targeting signal'; 'TM' indicates 'transmembrane domain'; 'C-C' indicates 'coiled-coil domain'. (B) (a) Human Mic60-GFP or Mic60-GFP plus Mic19-Flag were transiently expressed in 293T cells and immunoprecipitated with Flag resin, eluted protein samples were detected by western blot analysis using indicated antibodies; (b) HeLa cell lysates were used for co-immunoprecipitation assay by anti-Mic60 antibody, and the eluted protein samples were analyzed by western blot with anti-Mic19 or antiMic60 antibodies. (C) Interaction of human Mic60/Mitofilin with human Mic19/CHCHD3 in vitro analyzed by GST pull-down assay. Mic19-His, GST, GST-Mic60(35-200), GSTMic60(35-480) or GST-Mic60(371-590) was expressed in E. coli BL21, bacteria were then lysed and used for GST pull-down assay by using Pierce Glutathione Agarose

Figure 3 Mic60/Mitofilin is the substrate of mitochondrial protease Yme1L. (A) Mic60/Mitofilin interacts with Yme1L. (a) Human Mic60-GFP or Mic60-GFP plus Myc-tagged Yme1L-E600Q were transiently expressed in 293t cells and co-immunoprecipitated with 9E10 beads (anti-Myc resin), the protein samples were then analyzed by western blot with anti-GFP or anti-Myc antibodies; (b) HeLa cell lysates were used for co-immunoprecipitation by anti-Mic60 antibody, and the eluted protein samples were analyzed by western blot with anti-Yme1L or anti-Mic60 antibodies. (B) (a) The schematic representation of human Yme1L. 'MTS' indicates 'mitochondrial targeting signal'; 'TM' indicates 'transmembrane domain'. (b) Interaction of human Mic60/Mitofilin with human Yme1L in vitro determined by GST pull-down assay as described in 'Figure 2C'. (C) (a) Western blot analysis of Mic60/Mitofilin, Yme1L and GAPDH in control or shYme1L MEFs; (b) the protein levels of Mic60/Mitofilin and HSP60 in control or shYme1L MEFs were analyzed by immunostaining using indicated antibodies. The shYme1L MEFs were produced by infection with retrovirus generated by construct containing Yme1L short hairpin RNA and nuclear GFP. The MEFs expressing Nuclear GFP indicates shYme1L MEFs. (D) (a) Western blot analysis of Mic60/Mitofilin, Mic19/CHCHD3, Yme1L and GAPDH in control, shMic19 or shMic19 plus shYme1L MEFs; (b) the levels of Mic60/Mitofilin in control, shMic19 or shMic19 plus shYme1L MEFs were detected by immunostaining using antibody against Mic60 

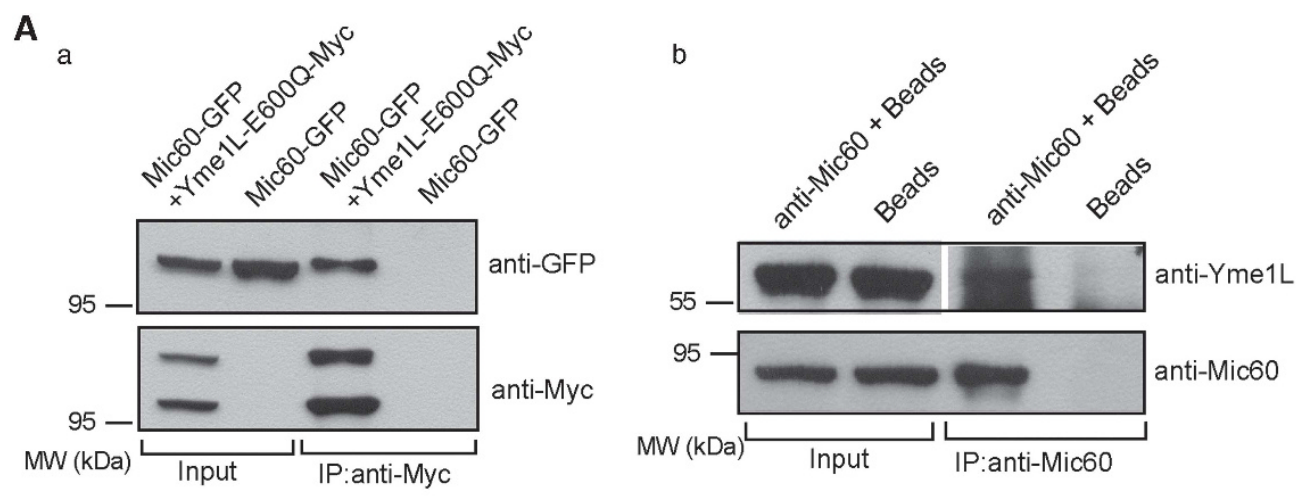

$B_{a}$
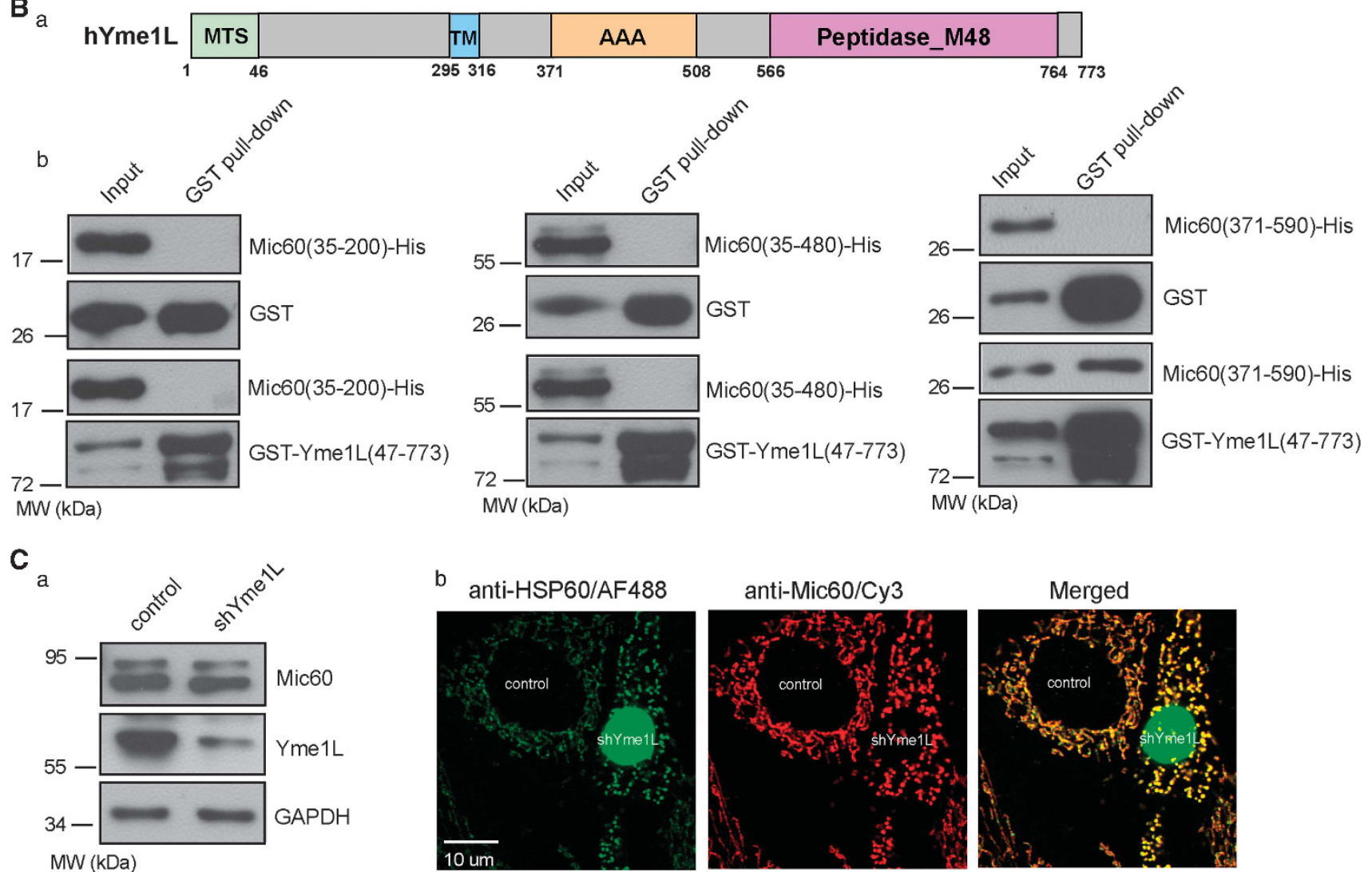

anti-Mic60/Cy3
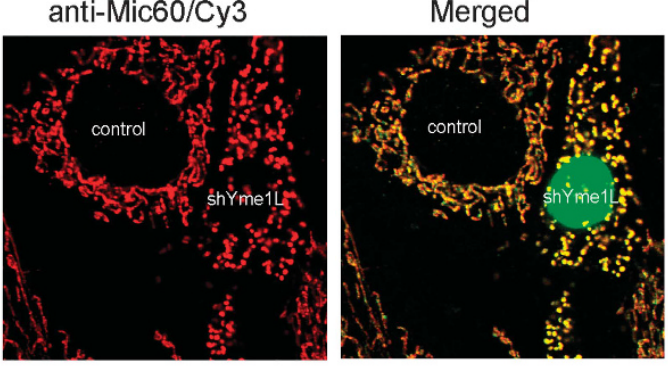

MEFs: control +shYme1L

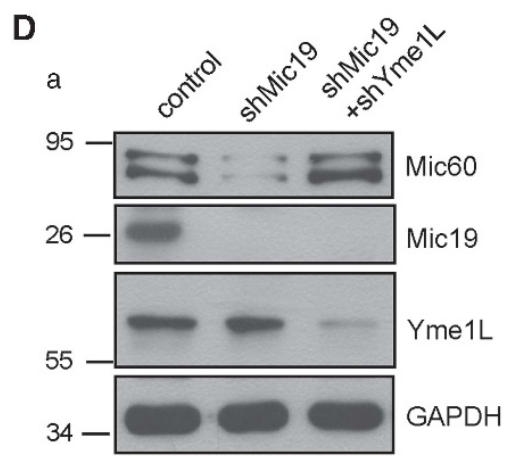

$\mathrm{MW}(\mathrm{kDa})$

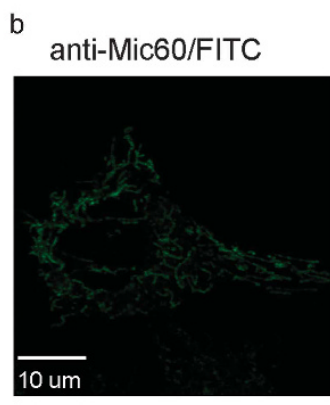

control
anti-Mic60/FITC

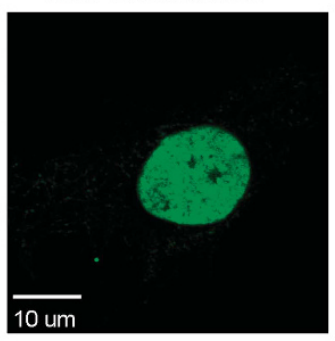

shMic19
anti-Mic60/FITC

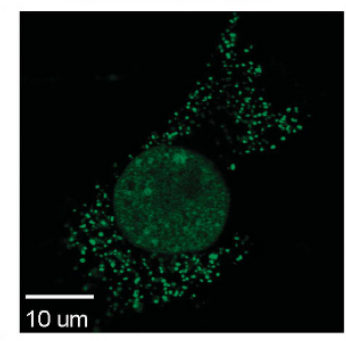

shMic19+shYme1L 
mitochondrial matrix targeted DsRed (mito-DsRed). WT MEFs exhibit largely tubular mitochondria; however, about $69 \%$ shMic60 MEFs showed enlarged spherical mitochondria, which are termed 'giant mitochondria' (Figure 4A). In addition, shMic19 leads to about 21\% MEFs containing 'giant mitochondria' but little shMic25 MEFs show

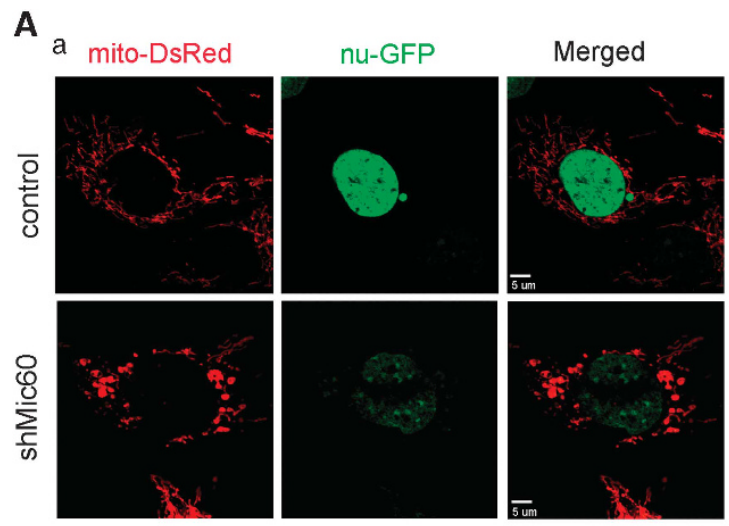

b

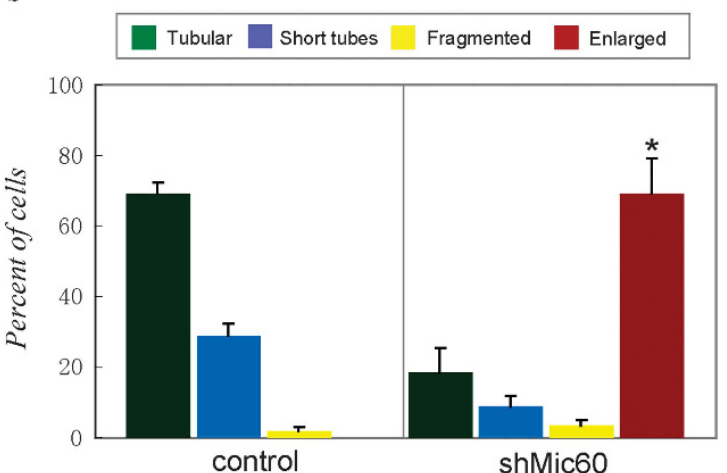

B

WT

shMic60

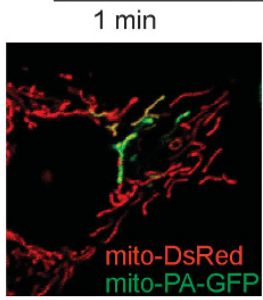

$20 \mathrm{~min}$

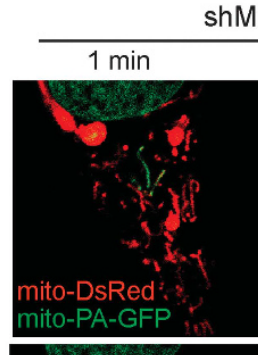

$20 \mathrm{~min}$
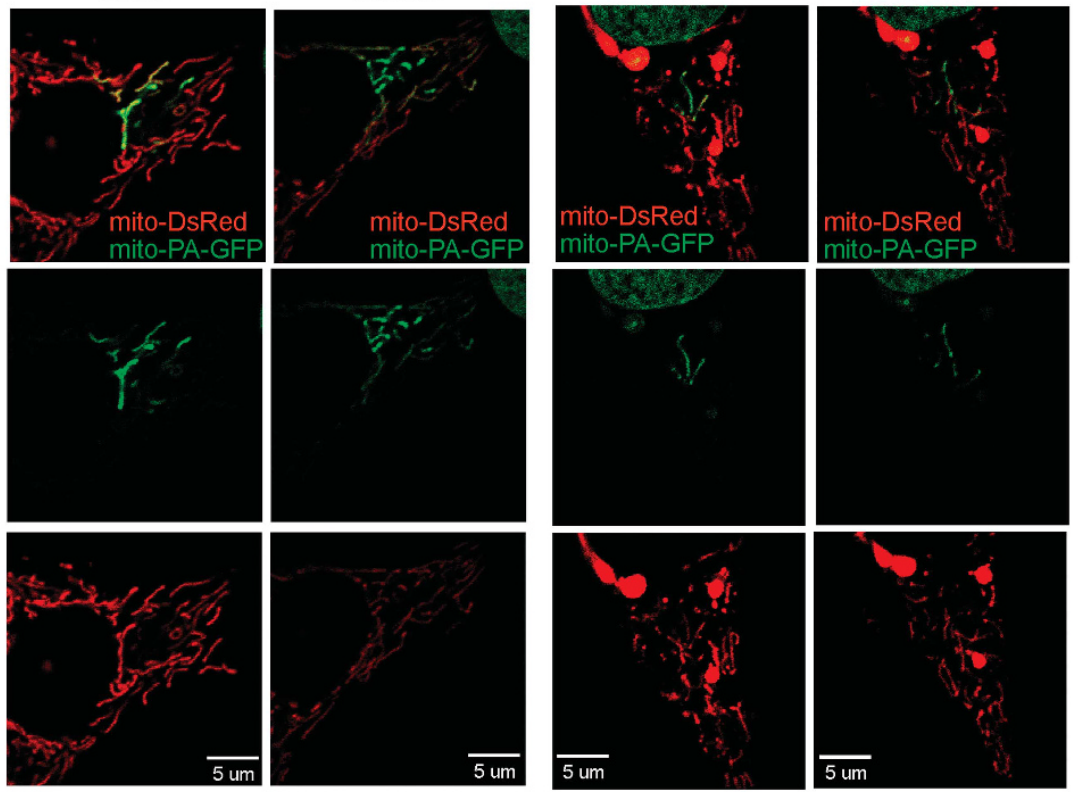

C
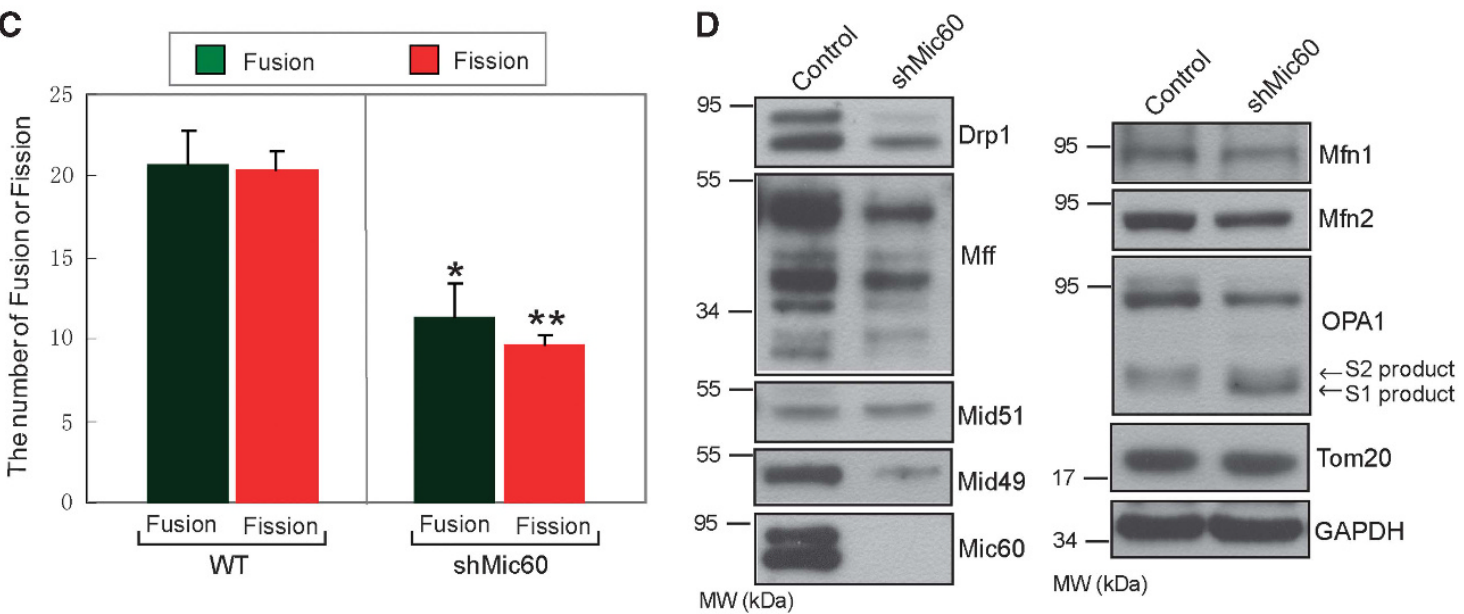
'giant mitochondria' (Supplementary Figure 4); interestingly, about 33\% Mic25 and Mic19 double knockdown MEFs contain 'giant mitochondria' (Supplementary Figure 4). These data suggest that Mic60/Mitofilin is crucial in maintaining mitochondrial shape in MEFs, and the integrity of MICOS complex is required for the regulation of mitochondrial morphology.

To directly assess the effect of shMic60 on mitochondrial fusion and fission activity, we stably expressed mito-DsRed and photoactivatible GFP (PA-GFP) targeted to mitochondrial matrix (mito-PA-GFP) in MEFs and performed PA-GFP assay. Within a single cell, we photoactivated a small subset of mitochondria by excitation and then tracked the mitochondrial fusion and fission events by time-lapse microscopy for about 20 min. In WT MEFs, mito-PA-GFP in mitochondria was found to be transferred to non-photoactivated mitochondria because of mitochondrial fusion (Figure 4B and Supplementary Movie 1). In shMic60 MEFs, photoactivated mitochondria could also transfer mito-PA-GFP to nonactivated mitochondria, but shMic60 MEFs displayed obviously less GFP fluorescence diffusion than WT MEFs (Figure 4B and Supplementary Movie 2); these results indicate that shMic60 leads to decreased mitochondrial fusion activity. We also tracked 10 photoactivated WT or shMic60 mitochondria labeled with mito-DsRed and mito-PA-GFP in MEFs, and quantified fusion and fission events within $20 \mathrm{~min}$. The quantitative data show that $10 \mathrm{WT}$ mitochondria proceed about 20.7 mitochondrial fusion and about 20.3 fission events per $20 \mathrm{~min}$; however, 10 shMic60 mitochondria undergo only about 11.3 fusion and 9.7 fission events within $20 \mathrm{~min}$ (Figure $4 \mathrm{C}$ ), suggesting that shMic60 leads to significant decreased mitochondrial fusion and fission. We conclude that shMic60 impairs mitochondrial dynamics.

In addition, we observed that shMic60 reduced the level of mitochondrial fusion and fission factors Mfn1, Mfn2, OPA1, Drp1, Mff and Mid49 (Figure 4D); moreover, shMic60 also led to the increased processing of OPA1 at S1 site but not at S2 site (Figure 4D). These results suggest that Mic60/Mitofilin is required for maintaining the stability of mitochondrial fusion and fission factors, and the disturbed mitochondrial dynamics induced by shMic60 is due to decreased mitochondrial fusion and fission factors.

Disorganized mtDNA nucleoids induced by Mic60/Mitofilin and Mic19/CHCHD3 deficiencies. To test the role of MICOS complex in the organization and distribution of mtDNA nucleoids, living MEFs expressing mito-DsRed were stained with SYBR Green I to visualize mtDNA nucleoids by confocal microscope. WT MEFs displayed normal-sized and normal-distributed mtDNA nucleoids ( $<1 \mu \mathrm{m}$; Figure $5 \mathrm{~A})$. However, shMic60 MEFs exhibited decreased amount of mtDNA nucleoids; importantly, shMic60 cells possessed some extremely enlarged nucleoids (>1 $\mu \mathrm{m}$; Figure $5 \mathrm{a}$ ). The quantitative data reveal that about $61.8 \%$ of shMic60 MEFs contain mtDNA nucleoids larger than $1 \mu \mathrm{m}$ in diameter (Figure 5B-a). Furthermore, almost all giant mitochondria induced by shMic60 contain enlarged mtDNA nucleiods (Figure 5a and Supplementary Figure 5A), and the short tubular in shMic60 MEFs harbor remarkably less number of mtDNA nucleiods than the similar length of short tubular in WT MEFs (Supplementary Figure 5), indicating that the enlarged mtDNA nucleoid is due to the clustering of nucleoids and the disorganized mtDNA nucleiods is highly associated with abnormal mitochondrial morphology.

To verify the role of Mic60/Mitofilin in organization of mtDNA nucleoids, we performed the recovery assay by expressing human Mic60/Mitofilin or Mic60/Mitofilin truncated mutants in shMic60 MEFs. The expression of human Mic60-Flag successfully inhibited the formation of giant mitochondria (data not shown) and enlarged mtDNA nucleoids induced by shMic60 in MEFs (Figure 5B), suggesting that human Mic60/ Mitofilin is functional in MEFs. Previous study showed that Mic60/Mitofilin was contained in purified HeLa mtDNA nucleoids $^{49}$ and embedded in mitochondrial inner membrane with its C-terminus facing mitochondrial intermembrane space. ${ }^{47,50}$ We then checked the effect of Mic60/Mitofilin $\mathrm{N}$-terminal truncates on the morphology of mtDNA nucleoids. Human Mic60(1-200)-Flag or Mic60(1-480)-Flag failed to rescue the morphology of mtDNA nucleoids in shMic60 MEFs (Figure 5B), suggesting that C-terminal of Mic60/Mitofilin is still required for maintenance of normal mtDNA nucleoids.

A recent study found that the deletion of Drp1 results in enlarged and clustered mitochondrial nucleoids; ${ }^{41}$ we showed that Mic60/Mitofilin deficiency caused a remarkable reduced Drp1 (Figure 4D). To investigate whether the clustering of mtDNA nucleoids induced by shMic60 is due to downregulation of Drp1, we overexpressed Drp1-YFP in WT or shMic60 MEFs and analyzed the morphology of mitochondria and mtDNA nucleoids. We observed that overexpression of Drp1-YFP results in increased mitochondrial fragmentation in WT cells and limit the formation of giant mitochondria in shMic60 MEFs (Supplementary Figure 6A); interestingly, Drp1-YFP expression partially inhibited the clustering of mtDNA nucleoids caused by shMic60 (Figure 5C), suggesting that Drp1 is associated with shMic60-induced clustering of mtDNA nucleoids. In addition, about $36 \%$ of cells still maintain enlarged mtDNA nucleoids in Drp1-YFP overexpressed shMic60 cells (Figure 5C), suggesting that shMic60-induced

Figure 4 Mitochondrial dynamics were impaired upon downregulation of Mic60/Mitofilin. (A) (a) The infected MEFs containing control or shMic60 were continue to cultured for 6 days, mitochondrial morphology in control and shMic60 MEFs expressing mito-DsRed (mitochondrial marker) and nu-GFP (nuclear marker) was then visualized by confocal microscope. (b) Mitochondrial morphology was counted according to the criteria detailed in Materials and Methods; bars represent means \pm S.D. of three independent experiments, and statistical significance were determined by Student's $t$-test $\left({ }^{*} P<0.05\right.$ versus control). Statistical significant differences are shown with asterisks. (B) Photoactivated mito-GFP-targeted mitochondria in WT or shMic60 MEFs expressing mito-PA-GFP and mito-DsRed and then were tracked by time-lapse microscopy for 20 min, mitochondrial fusion was assessed by the time-dependent dilution and redistribution of GFP fluorescence. (C) Comparison of mitochondrial fusion and fission between WT and shMic60 MEFs cell. Ten photoactivated mitochondria labeled with mito-PA-GFP were tracked by time-lapse microscopy for 20 min, and the number of mitochondrial fission and fusion events within 20 min were counted. Bars represent means \pm S.D. of three independent experiments. Statistical significance analyses were performed by using Student's t-test $\left({ }^{*} P<0.05\right.$ versus WT; ${ }^{*} P<0.05$ versus WT). (D) Levels of Drp1, Mff, Mid51, Mid49, Mfn1, Mfn2, OPA1 and Mic60/Mitofilin proteins in control and shMic60 MEFs were analyzed by western blot analysis using indicated antibodies. Tom20 and GAPDH were assayed as protein-loading control 
A
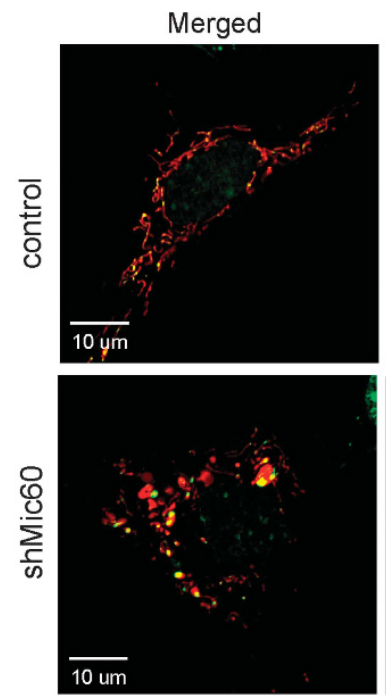

B

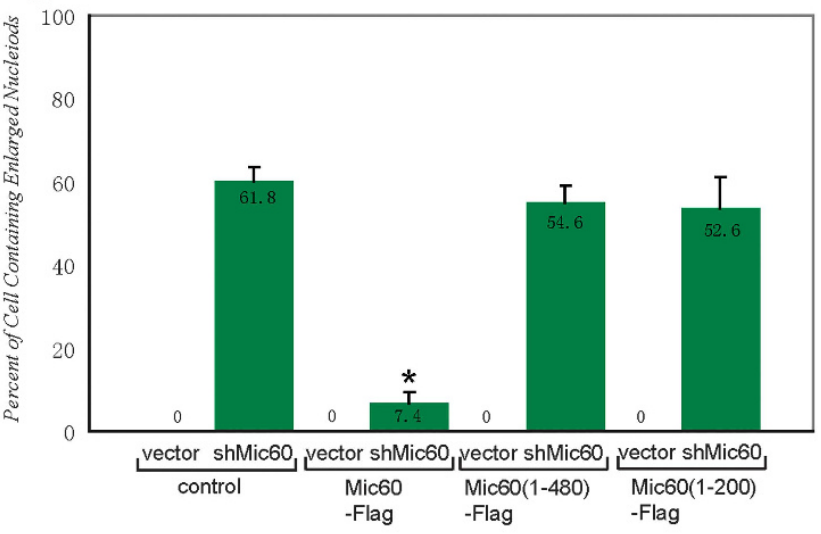

C a

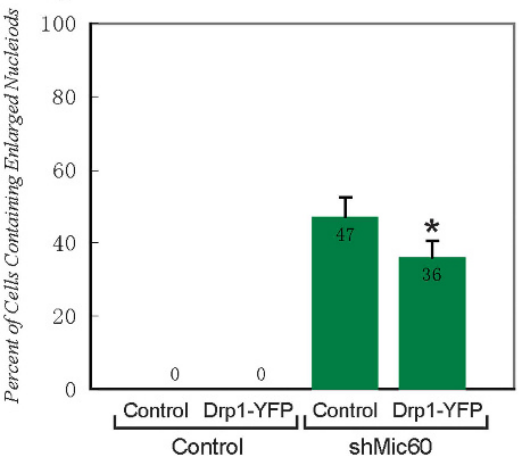

mito-DsRed
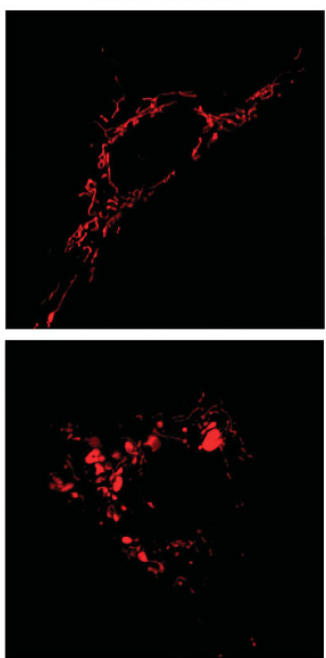

SYBR I
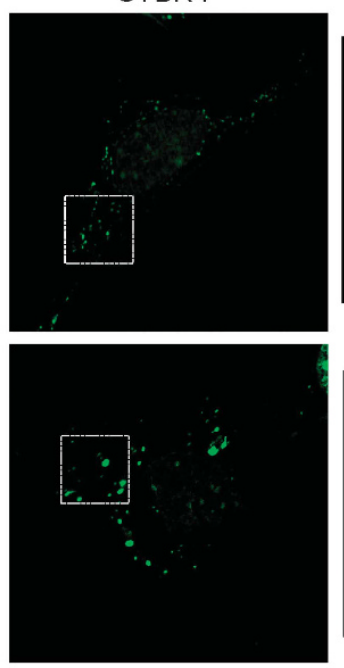
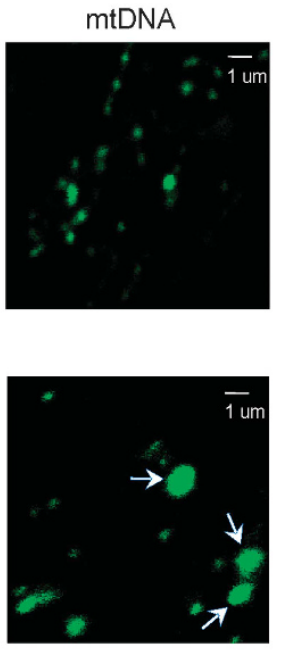

b

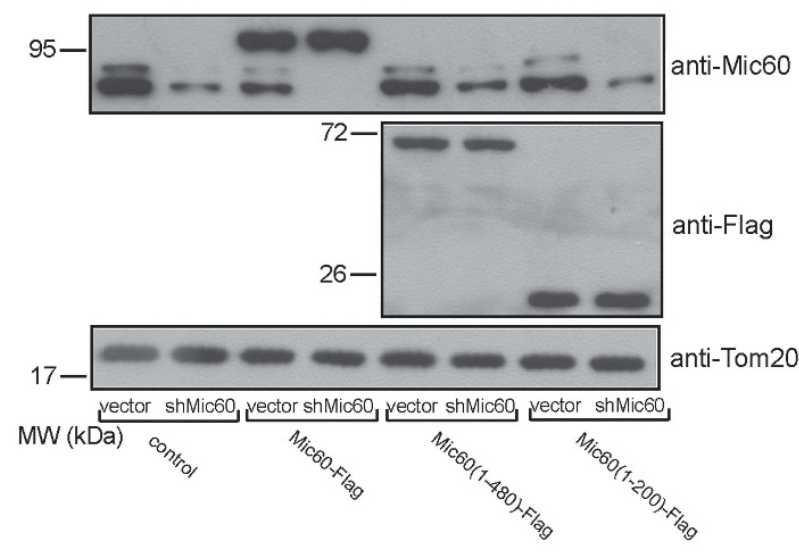

D a
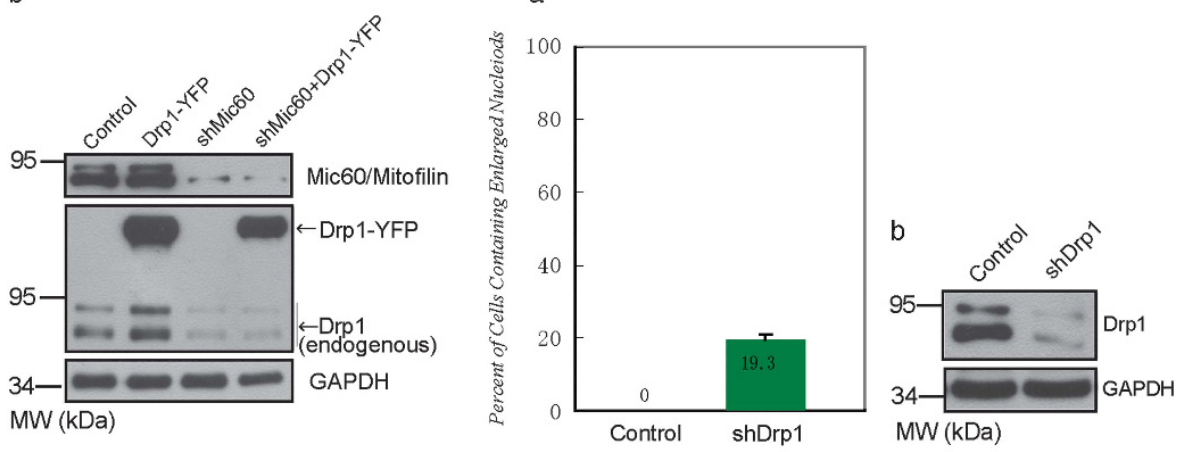

Figure 5 Downregulation of Mic60/Mitofilin leads to clustered mtDNA nucleoids independent on Drp1. (A) The infected MEFs containing control or indicated shRNA were cultured continuously for 6 days, with mtDNA nucleoids in control and shMic60 MEFs expressing mito-DsRed were stained with SYBR I, and the morphology of nucleoids was visualized by cofocal microscope. White arrow indicates enlarged mtDNA nucleoid. (B) (a) Control or shMic60 MEFs expressing human Mic60-Flag, Mic60(1-200)-Flag and Mic60(1-480)-Flag were stained with SYBR I, MEFs containing enlarged mtDNA nucleoids were then counted in each experiment. 100 MEFs were analyzed in each experiment. Bars represent means \pm S.D. of three independent experiments. Statistical significance analysis was performed by using Student's $t$-test $\left({ }^{\star} P<0.05\right.$ versus control shMic60 MEFs). (b) The lysates from the MEFs described in a were analyzed by western blot analysis with indicated antibodies. (C) (a) The morphology of mtDNA nucleoids in control and shMic60 MEFs expressing Drp1-GFP. MEFs containing enlarged mtDNA nucleoids were counted as described in B. (b) The cells lysates from the MEFs described in a were analyzed by western blot analysis using anti-Mic60, anti-Drp1 and anti-GAPDH antibodies. (D) (a) The morphology of mtDNA nucleoids in control and shDrp1 MEFs containing enlarged mtDNA nucleoids were counted as described in B. (b) Western blot analysis of control or shDrp1 MEFs using anti-Drp1 or anti-GAPDH antibody 
clustered mtDNA nucleoids is independent of Drp1, and demonstrating that Mic60/Mitofilin is a novel regulator of mtDNA nucleoid organization. In addition, about $60.3 \%$ of shMic60 MEFs display enlarged mtDNA nucleoids (Figure 5B); in contrast, only about $19.3 \%$ of shDrp1 MEFs display clustering of mtDNA nucleoids (Figure 5D), indicating that Mic60/Mitofilin is more critical than Drp1 in maintaining morphology of mtDNA nucleoids. It should be noted that shDrp1 also results in the formation of giant mitochondria in MEFs (Supplementary Figure 6B).

In addition, the size and distribution of mtDNA nucleoids were observed to be normal in shMic10 or shMic25 MEFs; however, about $21 \%$ shMic19 MEFs display enlarged clustered mtDNA nucleoids (Supplementary Figure 7A); and shMic19 caused a severe reduction of Mic60/Mitofilin (Supplementary Figure 7B); in contrast, shMic25 led to slight reduction of Mic60/Mitofilin, and shMic10 had no effect on stability of Mic60/Mitofilin (Supplementary Figure 7B). These results indicate that enlarged mtDNA nucleoids induced by shMic19 are probably due to the downregulation of Mic60/Mitofilin. We also observed more reduction of Mic60/ Mitofilin in Mic19 and Mic25 double knockdown MEFs than in shMic19 MEFs alone (Supplementary Figure 7B); importantly, the number of cells containing enlarged mtDNA nucleoids were increased to about 33\% upon Mic19 and Mic25 double knockdown (Supplementary Figure 7A), suggesting that the number of cells containing enlarged mtDNA nucleoids is positively correlated with the extent of downregulation of Mic60/Mitofilin. Taken together, comparing with other MICOS components, Mic60/Mitofilin is of central importance for maintenance of mtDNA nucleoids morphology.

Mic60 knockdown attenuates the transcription of mtDNA. To check whether mtDNA transcription were impaired upon disruption of MICOS complex, we assessed all 13 mitochondrial mRNA levels in WT and shMic60 MEFs by RT-qPCR. The mRNA levels for ND4L and COX2 were little changed in shMic60 MEFs (Figure 6a); however, all the other 11 mitochondrial mRNA levels were reduced upon Mic60/ Mitofilin depletion (Figure 6a); furthermore, the mRNA levels for ND2, ND4, ND5, COX1, ATP6 and ATP8 were significantly decreased in shMic60 MEFs (Figure 6a). These findings suggest that mtDNA transcription is attenuated upon Mic60/Mitofilin knockdown.

mtDNA nucleoid is composed of a set of mtDNA-binding proteins associated with mtDNA maintenance, replication and transcription. ${ }^{38,39}$ The core mtDNA nucleoid components center consists of transcription and replication factors, such as TFAM (the transcription factor $A$, mitochondrial), POLG (the mtDNA polymerase $\gamma$ ), Twinkle (the mtDNA helicase), mtSSB (the mitochondrial single-strand DNA-binding protein) ${ }^{38,39}$ and LONP1 (the Lon protease), were found to be an integral mtDNA nucleoid factor; and ATAD3, PHB1 and PHB2 (Prohibitin1 and Prohibitin2) provide molecular scaffolding to stabilize mtDNA nucleiod and promote mitochondrial translation; ${ }^{38,39}$ in addition, AFG3L2, a subunit of mitochondrial m-AAA protease, is associated with mtDNA nucleiod. ${ }^{38}$ Upon Mic60/Mitofilin depletion, the protein levels of TFAM and POLG were not altered (Figure 6b); however, LONP1 and AFG3L2 were decreased in shMic60 MEFs (Figure 6b); importantly, mtSSB, Twinkle, ATAD3, PHB1 and PHB2 were remarkably decreased upon Mic60/Mitofilin depletion (Figure 6b). These results indicate that LONP1, AFG3L2, mtSSB, Twinkle, ATAD3, PHB1 and PHB2 may be involved in disorganization of mtDNA nucleoids and decreased mtDNA transcription induced by Mic60/Mitofilin knockdown.

\section{Discussion}

In this study, we show that Mic60/Mitofilin, Mic19/CHCHD3 and Mic10/MINOS1 are key components of mammalian MICOS complex and required for MICOS assembly, and identified Yme1L regulates the stability of Mic60/Mitofilin. Furthermore, we report that the impaired MICOS complex by Mic60/Mitofilin knockdown induced a large number of giant mitochondria, and attenuates mitochondrial fusion and fission and disturbs mtDNA nucleoid organization (Figure 6c). Thus, our results suggest that the regulation of Mic60/Mitofilin proteostasis is essential for MICOS assembly and provides a novel clue to link mitochondrial dynamics and mitochondrial nucleoid organization.

The detailed molecular mechanism of mitochondrial dynamics is still largely unknown, although the key factors for mitochondrial dynamics are already identified. ${ }^{51}$ In the present study, we found that the disassembly of MICOS complex induced by shMic60 leads to remarkable reduced mitochondrial fusion and fission due to decreased Drp1, Mff, Mid49, OPA1 and Mfn2 (Figures 4C and D). Our findings indicate that Mic60/Mitofilin may function as a mediator linking mitochondrial outer membrane and inner membrane fusion or fission. Thus, Mic60/Mitofilin is a novel regulator of mitochondrial dynamics. Interestingly, lots of spherical giant mitochondria were observed in Mic60/Mitofilin-depleted MEFs (Figure 4A). In mammals, the proteins regulating giant mitochondria formation are still not identified, although giant mitochondria were reported to be normally observed in the aged or some diseased mammalian cells. ${ }^{29-33}$ Our data suggest that Mic60/Mitofilin is a key factor in regulating the formation of giant mitochondria in mammals. In addition, it will be interesting in the future studies to demonstrate how downregulation of Mic60/Mitofilin destabilizes some mitochondrial fusion or fission factors.

The mechanism of organization, distribution and dynamics of mtDNA nucleoids are poorly understood in mammals. Recently, mitochondrial fusion and fission factors have been shown to influence the organization of mtDNA nucleoids. ${ }^{41,52,53}$ In the present study, we found that Mic60/Mitofilin has an important role in the maintaining of mtDNA nucleoid organization and function in mammals (Figures 5B and 6a). Thus, an important question is how Mic60/Mitofilin regulates the distribution and size of mtDNA nucleoids? Mic60/Mitofilin localizes in close vicinity of mtDNA nucleoids; ${ }^{49}$ and we found that the shMic60 results in the loss of mitochondrial cristae junctions (Figure 1A); in addition, Drp1 overexpression partially reduces the clustering of mtDNA nucleoids caused by shMic60 (Figure $5 \mathrm{C}$ ). Thus, we provide a hypothesis that mitochondrial cristae junctions connect cristae to inner boundary membrane to maintain proper internal membrane compartments where mtDNA nucleoids are located; and proper mitochondrial fission is required to 

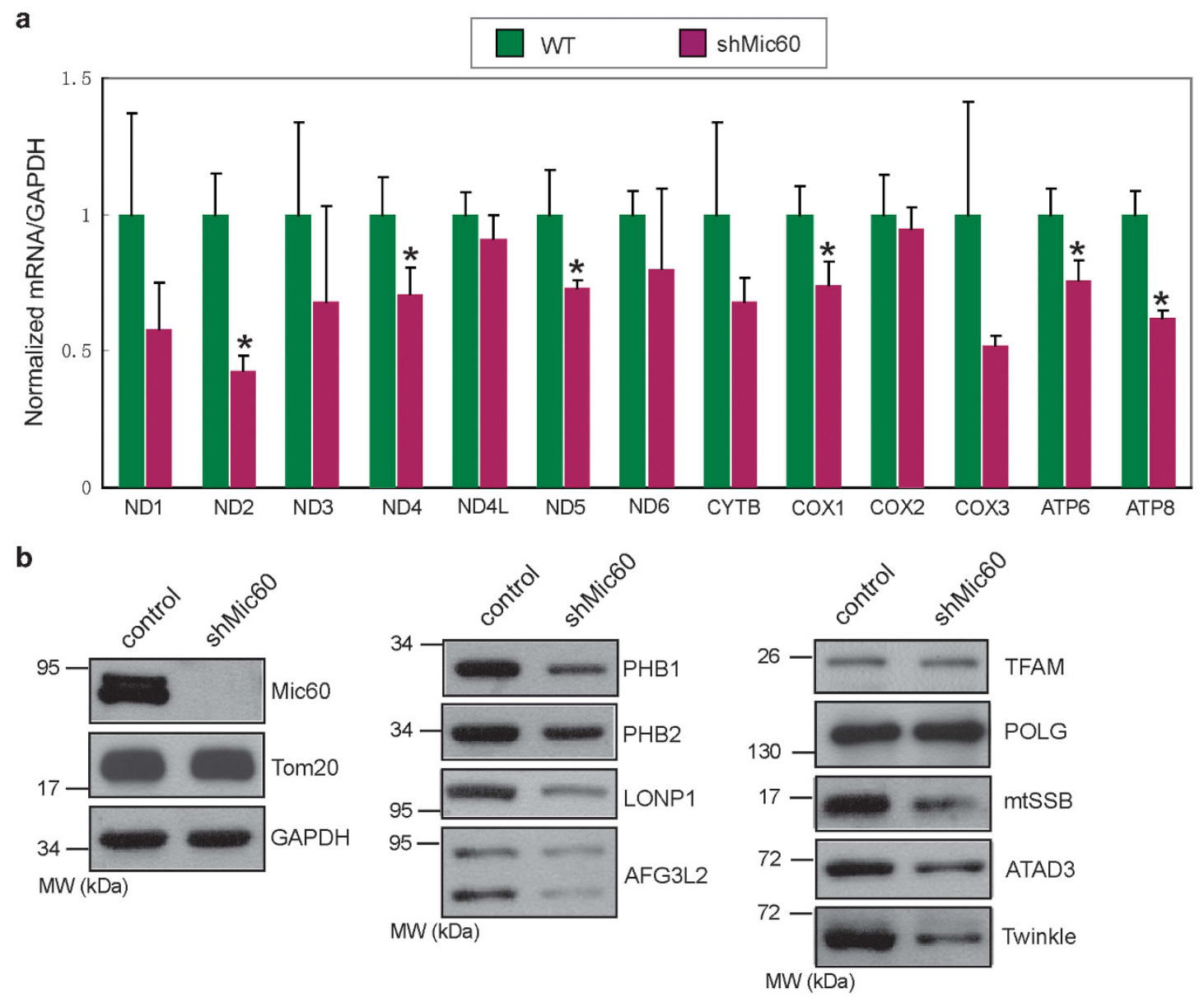

c

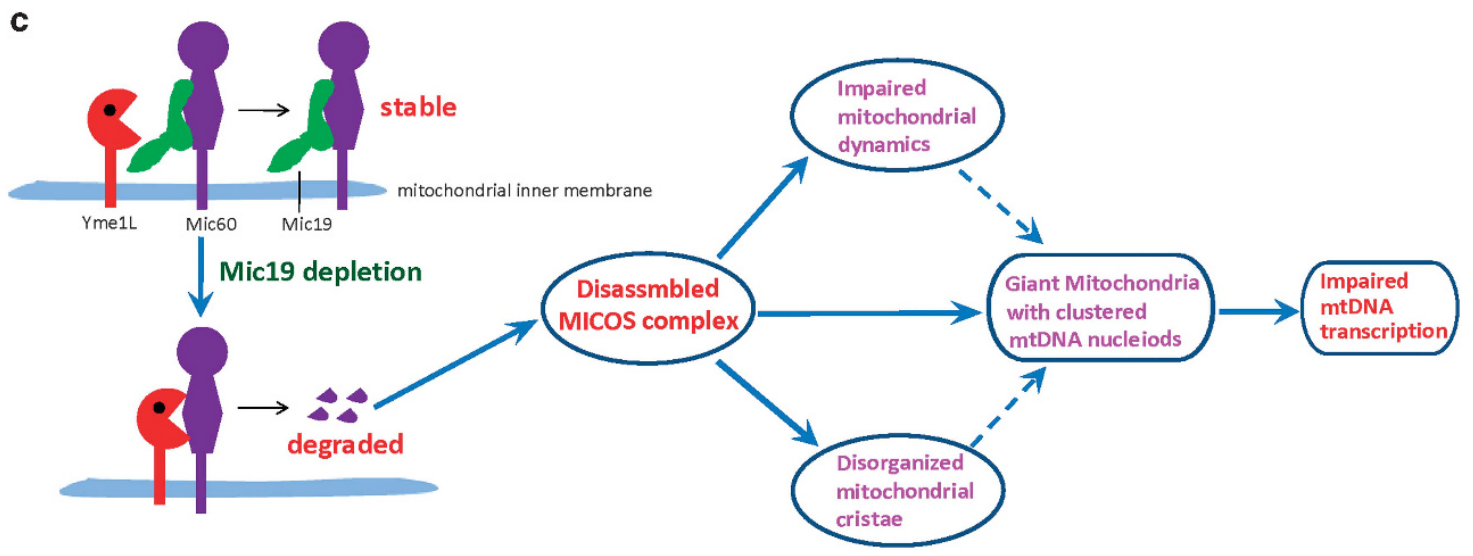

Figure 6 Mic60/Mitofilin reduction attenuates mtDNA transcription. (a) 13 mitochondrial mRNA levels in WT or shMic60 MEFs were analyzed by RT-qPCR. Error bars are means \pm S.D. of three independent experiments. Statistical significance analysis was performed by using Student's $t$-test $\left({ }^{\star} P<0.05\right.$ versus WT). (b) Cell lysates of WTor shMic60 MEFs were analyzed by western blot analysis with antibodies against indicated mtDNA nucleoids associated with proteins. (c) The model of Mic60/Mitofioin homeostasis and its effect on mitochondrial dynamics and mtDNA nucleoid organization. Under normal condition, Mic60/Mitofilin is stabilized by Mic19/CHCHD3 by direct interaction. Upon Mic19/CHCHD3 reduction, Mic60/Mitofilin is degraded by mitochondrial protease Yme1L. Furthermore, Mic60/Mitofilin downregulation causes abnormal cristae structure, impaired mitochondrial dynamics and enlarged mtDNA nucleiods; therefore, mtDNA transcription is attenuated

separate mtDNA nucleoids. The loss of mitochondrial cristae junctions resulting from depletion of Mic60/Mitofilin breaks the limitation of internal membrane compartments for distribution of mtDNA nucleoids and leads to clustering of mtDNA nucleoids; in addition, decreased mitochondrial fission in shMic60 cells may inhibit the separation of adjacent mtDNA nucleoids and result in clustering. Taken together, our findings demonstrate that mitochondrial dynamics and inner membrane organization, linked by Mic60/Mitofilin, are required for distribution, organization and function of mtDNA nucleoids.

Alterations of mitochondrial architecture and dynamics are linked to several human diseases. ${ }^{27,28,54,55}$ Our study showed 
that Mic60/Mitofilin homeostasis regulates mitochondrial cristae structure, mitochondrial dynamics and nucleoid organization (Figure 6c), indicating that the homeostasis of Mic60/ Mitofilin and other MICOS components may be associated with some human diseases. Actually, impaired Mic60/Mitofilin homeostasis has been described in a set of pathologies associated with cardiomyopathy, brain development, function and degeneration. ${ }^{42-46,56}$ Future studies will have to uncover the role and the molecular mechanisms of Mic60/Mitofilin and other MICOS subunits in the pathogenesis of the diseases.

\section{Materials and Methods}

Cell culture and transfection. MEFs, Hela and 293T cells were cultured in Dulbecco's modified Eagle's medium supplemented with $10 \%$ fetal bovine serum, $1 \%$ penicillin/streptomycin and $1 \%$ L-glutamine at $37^{\circ} \mathrm{C}$ with $5 \% \mathrm{CO}_{2}$. Lipofectamine 2000 and Opti-MEM I (Invitrogen, Carlsbad, CA, USA) were used for transient transfection according to the manufacturer's protocol.

Western blot, Co-IP and GST-pulldown assay. Western blot and co-IP were performed as we have described previously. ${ }^{57}$ Cells were lysed in a Triton X-100based lysis buffer (1\% Triton X-100, 10\% glycerol, $20 \mathrm{mM}$ Tris, $150 \mathrm{mM} \mathrm{NaCl}$, pH7.4, $2 \mathrm{mM}$ EDTA, protease inhibitor mixture). GST- or His-tagged proteins were expressed in E. coli BL21 and induced with $1 \mathrm{mM} \mathrm{IPTG.} \mathrm{Bacteria} \mathrm{were} \mathrm{then} \mathrm{lysed} \mathrm{and} \mathrm{centrifuged,}$ the supernatants were mixed with Pierce Glutathione Agarose (Thermo Scientific, Waltham, MA, USA) for $4 \mathrm{~h}$ at $4^{\circ} \mathrm{C}$. Beads were collected by $2000 \times g$ centrifugation and washed five times with PBS. The samples were analyzed by western blot analysis.

Confocal microscopy and image processing. Confocal microscopy (FV1000) was performed with an Olympus IX81 microscope with a 100 x UPL SAPO $\mathrm{NA}=1.4$ oil objective (Olympus Corporation, Tokyo, Japan). MEFs or HeLa cells were cultured on coverslips and fixed with 10\% formalin. The FV10-ASW 3.0 software (Olympus Corporation) was used for image processing and analysis. Photoactivable GFP assay was performed as described previously. ${ }^{21}$ To determine mitochondrial morphology, cells were randomly selected for quantitative analysis and visually scored into four classifications ('Tubular', 'short Tubes', 'Fragmented' and 'Enlarged').

Electron microscopy. MEFs were fixed and prepared as we have described previously. ${ }^{21}$ The stained sections were imaged onto negatives using a Jeol electron microscope (Joel Ltd, Tokyo, Japan) operated at $80 \mathrm{kV}$.

BN-PAGE analysis. Isolated mitochondrial were re-suspended in BN-lysis buffer $(20 \mathrm{mM}$ bis-Tris, $500 \mathrm{mM} \varepsilon$-aminocaproic acid, $20 \mathrm{mM} \mathrm{NaCl}, 2 \mathrm{mM}$ EDTA, $10 \%$ glycerol, $1 \mathrm{mM}$ PMSF and $1 \mathrm{x}$ cocktail) on ice, and lysed by the addition of $0.5 \%$ digitonin for $2 \mathrm{~h}$. The samples were separated on $4-15 \%$ polyacrylamide gels and repeated loading for three times.The gel was transferred to PVDF and immunoblotted with the anti-Mic60/Mitofilin, anti-Mic10/MINOS1 antibody or antiMic19/CHCHD3 antibody, respectively.

Quantitative PCR. RNA from WT or shMic60 MEFs cells were prepared using TRIzol Reagent (Invitrogen) according to the manufacturer's instructions. Genome removed by RNase-free DNase I (Thermo Scientific). Reverse transcription was performed using the RevertAid First Strand CDNA Synthesis Kit (Thermo Scientific). RT-qPCR was performed with SsoAdvanced SYBR Green Supermix (Bio-Rad, Hercules, CA, USA) on the Rotor Gene 6000 Real-time Analyzer (Biolabo Scientific Instruments SA, Châtel-St-Denis, Switzerland). GAPDH was served as an internal control. The primer sets for mitochondrial transcripts were used as described previously. ${ }^{58}$

\section{Conflict of Interest}

The authors declare no conflict of interest.

Acknowledgements. We thank Dr Quan Chen (Institute of Zoology, Chinese Academy of Sciences) and David Chan (California Institute of Technology) for communicating results before publication. This work is supported by the National Key Basic Research Program of China (2013CB531200), National Natural Science
Foundation of China (31471264, 31171357 and 81101525), Hubei Natural Science Foundation (2014CFA023) and the Large-scale Instrument and Equipment Sharing Foundation of Wuhan University.

1. Chacinska A, Koehler CM, Milenkovic D, Lithgow T, Pfanner N. Importing mitochondrial proteins: machineries and mechanisms. Cell 2009; 138: 628-644.

2. Neupert W, Herrmann JM. Translocation of proteins into mitochondria. Annu Rev Biochem 2007; 76: 723-749.

3. Frey TG, Mannella CA. The internal structure of mitochondria. Trends Biochem Sci 2000; 25 319-324.

4. Zick M, Rabl R, Reichert AS. Cristae formation - linking ultrastructure and function of mitochondria. Biochim Biophys Acta 2009; 1793: 5-19.

5. Vogel F, Bornhövd C, Neupert W, Reichert AS. Dynamic subcompartmentalization of the mitochondrial inner membrane. J Cell Biol 2006; 175: 237-247.

6. Tamura Y, HaradaY, Shiota T, Yamano K, Watanabe K, Yokota M et al. Tim23-Tim50 pair coordinates functions of translocators and motor proteins in mitochondrial protein import. $J$ Cell Biol 2009; 184: 129-141.

7. Chacinska A, Lind M, Frazier AE, Dudek J, Meisinger C, Geissler A et al. Mitochondrial presequence translocase: switching between TOM tethering and motor recruitment involves Tim21 and Tim17. Cell 2005; 120: 817-829.

8. Gilkerson RW, Selker JM, Capaldi RA. The cristal membrane of mitochondria is the principal site of oxidative phosphorylation. FEBS Lett 2003; 546: 355-358.

9. Renken C, Siragusa G, Perkins G, Washington L, Nulton J, Salamon P et al. A thermodynamic model describing the nature of the crista junction: a structural motif in the mitochondrion. J Struct Biol 2002; 138: 137-144.

10. Harner M, Körner C, Walther D, Mokranjac D, Kaesmacher J, Welsch U et al. The mitochondrial contact site complex, a determinant of mitochondrial architecture. EMBO J 2011; 30: 4356-4370.

11. Hoppins S, Collins SR, Cassidy-Stone A, Hummel E, Devay RM, Lackner LL et al. A mitochondrial-focused genetic interaction map reveals a scaffold-like complex required for inner membrane organization in mitochondria. J Cell Biol 2011; 195: 323-340.

12. Von der Malsburg K, Müller JM, Bohnert M, Oeljeklaus S, Kwiatkowska P, Becker T et al. Dual role of mitofilin in mitochondrial membrane organization and protein biogenesis. Dev Cell 2011; 21: 694-70.

13. Alkhaja AK, Jans DC, Nikolov M, Jans DC, Nikolov M, Vukotic M et al. MINOS1 is a conserved component of mitofilin complexes and required for mitochondrial function and cristae organization. Mol Biol Cell 2012; 23: 247-257.

14. Pfanner N, van der Laan M, Amati P, Capaldi RA, Caudy AA, Chacinska A et al. Uniform nomenclature for the mitochondrial contact site and cristae organizing system. J Cell Biol 2014; 204: 1083-1086.

15. Xie J, Marusich MF, Souda P, Whitelegge J, Capaldi RA. The mitochondrial inner membrane protein mitofilin exists as a complex with SAM50, metaxins 1 and 2, coiled-coil-helix coiledcoil-helix domain-containing protein 3 and 6 and DnaJC11. FEBS Lett 2007; 581: 3545-3549.

16. Darshi M, Mendiola VL, Mackey MR, Murphy AN, Koller A, Perkins GA et al. ChChd3, an inner mitochondrial membrane protein, is essential for maintaining crista integrity and mitochondrial function. J Biol Chem 2011; 286: 2918-2932.

17. An J, Shi J, He Q, Lui K, Liu Y, Huang Y et al. CHCM1/CHCHD6, novel mitochondrial protein linked to regulation of mitofilin and mitochondrial cristae morphology. J Biol Chem 2012; 287 $7411-7426$.

18. Weber TA, Koob S, Heide H, Wittig I, Head B, van der Bliek A et al. APOOL is a cardiolipinbinding constituent of the Mitofilin/MINOS protein complex determining cristae morphology in mammalian mitochondria. PLoS One 2013; 8: e63683.

19. Baker MJ, Tatsuta T, Langer T. Quality control of mitochondrialproteostasis. Cold Spring Harb Perspect Biol 2011; 3 pii: a007559.

20. Song Z, Chen H, Fiket M, Alexander C, Chan DC. OPA1 processing controls mitochondrial fusion and is regulated by mRNA splicing, membrane potential, and Yme1L. J Cell Biol 2007; 178: 749-755.

21. Ruan Y, Li H, Zhang K, Jian F, Tang J, Song Z. Loss of Yme1L perturbates mitochondrial dynamics. Cell Death Dis 2013; 4: e896.

22. Stiburek L, Cesnekova J, Kostkova O, Fornuskova D, Vinsova K, Wenchich L et al. YME1L controls the accumulation of respiratory chain subunits and is required for apoptotic resistance, cristae morphogenesis, and cell proliferation. Mol Biol Cell 2012; 23: 1010-1023.

23. Youle RJ, van der Bliek AM. Mitochondrial fission, fusion, and stress. Science 2012; 337 1062-1065.

24. Song Z, Ghochani M, McCaffery JM, Frey TG, Chan DC. Mitofusins and OPA1 mediate sequential steps in mitochondrial membrane fusion. Mol Biol Cell 2009; 20: 3525-3532.

25. Losón OC, Song Z, Chen H, Chan DC. Fis1, Mff, MiD49, and MiD51 mediate Drp1 recruitment in mitochondrial fission. Mol Biol Cell 2013; 24: 659-667.

26. Chan DC. Fusion and fission: interlinked processes critical for mitochondrial health. Annu Rev Genet 2012; 46: 265-287.

27. Zuchner S, Mersiyanova IV, Muglia M, Bissar-Tadmouri N, Rochelle $\mathrm{J}$ et al. Mutations in themitochondrial GTPasemitofusin 2 cause Charcot-Marie-Toothneuropathy type 2 A. Nat Genet 2004; 36: 449-451. 
28. Alexander C, Votruba M, Pesch UE, Thiselton DL, Mayer S et al. OPA1, encoding a dynaminrelated GTPase, is mutated in autosomal dominant optic atrophy linked to chromosome 3q28. Nat Genet 2000; 26: 211-215.

29. Yen WL, Klionsky DJ. How to livelong and prosper: autophagy, mitochondria, and aging Physiology (Bethesda) 2008; 23: 248-262.

30. Kanzaki Y, Terasaki F, Okabe M, Otsuka K, Katashima T, Fujita S et al. Giant mitochondria in the myocardium of a patient with mitochondrial cardiomyopathy: transmission and 3-dimensional scanning electron microscopy. Circulation 2010; 121: 831-832.

31. Coleman R, Silbermann M, Gershon D. Reznick AZ.Giant mitochondria in the myocardium of aging and endurance-trained mice. Gerontology 1987; 33: 34-39.

32. Tandler B, Dunlap M, Hoppel CL, Hassan M. Giant mitochondria in a cardiomyopathic heart. Ultrastruct Pathol 2002; 26: 177-183.

33. Terman A, Dalen H, Eaton JW, Neuzil J, Brunk UT. Aging of cardiac myocytes in culture: oxidative stress, lipofuscin accumulation, and mitochondrial turnover. Ann N Y Acad Sci 2004; 1019: 70-77.

34. Burgess SM, Delannoy M, Jensen RE. MMM1encodes a mitochondrial outer membrane protein essential for establishing and maintaining the structure of yeast mitochondria. J Cell Biol 1994; 126: 1375-1391.

35. Berger KH, Sogo LF, Yaffe MP. Mdm12p a component required for mitochondrial inheritance that is conserved between budding and fission yeast. J Cell Biol 1997; 136: 545-553.

36. Sogo LF, Yaffe MP. Regulation of mitochondrial morphology and inheritance by Mdm10p, a protein of the mitochondrial outer membrane. J Cell Biol 1994; 126: 1361-1373.

37. Dimmer KS, Jakobs S, Vogel F, Altmann K, Westermann B. Mdm3 1 and Mdm32 are inner membrane proteins required for maintenance of mitochondrial shape and stability of mitochondrial DNA nucleoids in yeast. J Cell Biol 2005; 168: 103-115.

38. Hensen F, Cansiz S, Gerhold JM, Spelbrink JN. To be or not to be a nucleoid protein: a comparison of mass-spectrometry based approaches in the identification of potential mtDNA-nucleoid associated proteins. Biochimie 2014; 100: 219-226.

39. Gilkerson R, Bravo L, Garcia I, Gaytan N, Herrera A, Maldonado A et al. The mitochondrialnucleoid: integratingmitochondrialDNA into cellular homeostasis. Cold Spring Harb Perspect Biol 2013; 5: a011080.

40. Gilkerson RW. Mitochondrial DNA nucleoids determine mitochondrial genetics and dysfunction. Int J Biochem Cell Biol 2009; 41: 1899-1906.

41. Ban-Ishihara R, Ishihara T, Sasaki N, Mihara K, Ishihara N. Dynamics of nucleoid structure regulated by mitochondrial fission contributes to cristae reformation and release of cytochrome c. Proc Natl Acad Sci USA 2013;.110: 11863-11868.

42. Bernert G, FountoulakisM, Lubec G. Manifolddecreased protein levels of matrin 3, reduced motor proteinHMP and hlark in fetal Down's syndrome brain. Proteomics 2002; 2: 1752-1757.

43. Baseler WA, Dabkowski ER, Williamson CL, Croston TL, Thapa D, Powell MJ et al. Proteomicalterations of distinctmitochondrial subpopulations in the type 1 diabetic heart: contribution of protein import dysfunction. Am J Physiol Regul Integr Comp Physiol 2011; 300: R186-R200.

44. Kim N, Lee Y, Kim H, Joo H, Youm JB, Park WS et al. Potential biomarkers for ischemic heart damage identified in mitochondrial proteins by comparative proteomics. Proteomics 2006; 6 : 1237-1249.

45. O'Connell K, Ohlendieck K. Proteomic DIGE analysis of the mitochondria-enriched fraction from aged rat skeletal muscle. Proteomics 2009; 9: 5509-5524.

46. Guo Y, Darshi M, Ma Y, Perkins GA, Shen Z, Haushalter KJ et al. Quantitative proteomic and functional analysis of liver mitochondria from high fat diet (HFD) diabetic mice. Mol Cell Proteomics 2013; 12: 3744-3758.

47. John GB, Shang Y, Li L, Renken C, Mannella CA, Selker JM et al. The mitochondrial inner membrane protein mitofilin controls cristae morphology. Mol Biol Cell 2005; 16: 1543-1554.

48. Frezza C, Cipolat S, Martins de Brito O, Micaroni M, Beznoussenko GV, Rudka T. OPA1 controls apoptotic cristae remodeling independently from mitochondrial fusion. Cell 2006; 126: 177-189.

49. Wang Y, Bogenhagen DF. Human mitochondrial DNA nucleoids are linked to protein folding machinery and metabolic enzymes at the mitochondrial inner membrane. J Biol Chem 2006; 281: 25791-25802.

50. Odgren PR, Toukatly G, Bangs PL, Gilmore R, Fey EG. Molecular characterization of mitofilin (HMP), a mitochondria-associated protein with predicted coiled coil and intermembrane space targeting domains. J Cell Sci 1996; 109: 2253-2264.

51. Hoppins S. The regulation of mitochondrial dynamics. Curr Opin Cell Biol 2014; 29: 46-52.

52. Elachouri G, Vidoni S, Zanna C, Pattyn A, Boukhaddaoui H, Gaget K et al. OPA1 links human mitochondrial genome maintenance to mtDNA replication and distribution. Genome Res 2011; 21: 12-20.

53. Itoh K, Tamura $\mathrm{Y}$, lijima M, Sesaki H. Effects of Fcj1-Mos1 and mitochondrial division on aggregation of mitochondrial DNA nucleoids and organelle morphology. Mol Biol Cell 2013; 24: 1842-1851.

54. Liesa M, Palacín M, Zorzano A. Mitochondrial dynamics in mammalian health and disease. Physiol Rev 2009; 89: 799-845.

55. Zerbes RM, van der Klei IJ, Veenhuis M, Pfanner N, van der Laan M, Bohnert M. Mitofilin complexes: conserved organizers of mitochondrial membrane architecture. Biol Chem 2012; 393: $1247-1261$.

56. Zhang Y, Xu J, Luo YX, An XZ, Zhang R, Liu G et al. Overexpression of mitofilin in the mouse heart promotes cardiac hypertrophy in response to hypertrophic stimuli. Antioxid Redox Signal 2014; 21: 1693-1707.

57. Zhang K, Li H, Song Z. Membrane depolarization activates the mitochondrial protease OMA1 by stimulating self-cleavage. EMBO Rep 2014; 15: 576-585.

58. Zhang X, Zuo X, Yang B, Li Z, Xue Y, Zhou Y et al. MicroRNA directly enhances mitochondrial translation during muscle differentiation. Cell 2014; 158: 607-619.

Supplementary Information accompanies this paper on Cell Death and Differentiation website (http://www.nature.com/cdd) 